\title{
Ligand-induced conformational change of Insulin- regulated aminopeptidase: insights on catalytic mechanism and active site plasticity
}

\author{
Anastasia Mpakali1, Emmanuel Saridakis' ${ }^{1}$, Karl Harlos², Yuguang Zhao ${ }^{2}$, \\ Paraskevi Kokkala ${ }^{3}$, Dimitris Georgiadis ${ }^{3}$, Petros Giastas ${ }^{1}$, Athanasios \\ Papakyriakou ${ }^{1}$ and Efstratios Stratikos ${ }^{1, *}$ \\ ${ }^{1}$ National Center for Scientific Research Demokritos, Agia Paraskevi, Athens \\ 15310, Greece \\ ${ }^{2}$ Division of Structural Biology, Wellcome Trust Centre for Human Genetics, \\ Oxford University, Oxford OX3 7BN, United Kingdom \\ ${ }^{3}$ Department of Chemistry, University of Athens, Athens 15771, Greece
}

\section{ABSTACT}

Insulin-regulated aminopeptidase (IRAP) is an enzyme with several important biological functions that is known to process a large variety of different peptidic substrates although the mechanism behind this wide specificity is not clearly understood. We describe a crystal structure of IRAP in complex with a recently developed bioactive and selective inhibitor at $2.53 \AA$ resolution. In the presence of this inhibitor the enzyme adopts a novel conformation in which domains II and IV are juxtaposed, forming a hollow structure that excludes external solvent access to the catalytic center. A loop adjacent to the enzyme's GAMEN motif undergoes structural reconfiguration, allowing the accommodation of bulky inhibitor side-chains. Atomic interactions between the inhibitor and IRAP that are unique to this conformation can explain the strong selectivity compared to homologous aminopeptidases ERAP1 and ERAP2. This conformation provides insight on IRAP's catalytic cycle and reveals significant active site plasticity that may underlie its substrate permissiveness. 


\section{INTRODUCTION}

Insulin-regulated aminopeptidase (IRAP, also known as Leucyl-cystinyl aminopeptidase, placental leucine aminopeptidase and oxytocinase, EC 3.4.11.3) is a transmembrane zinc aminopeptidase that is tasked with several biological functions which include the generation of antigenic peptides for crosspresentation, the regulation of trafficking of the glucose transporter type 4, the control of oxytocin levels in pregnancy and the regulation of brain oxytocin and vasopressin levels ${ }^{1}$. IRAP also has a specific binding site for angiotensin IV ${ }^{2}$. The soluble extra-cellular domain of IRAP carries the aminopeptidase activity and is highly homologous to two other intracellular aminopeptidases that are tasked with the generation of antigenic peptides, namely ER aminopeptidases 1 and 23 . Accordingly, IRAP has been implicated in a distinct intracellular pathway that generates antigenic peptides for cross-presentation by dendritic cells ${ }^{4}$. The important biological functions of IRAP have made this enzyme a target for developing small-molecular weight inhibitors that aim to regulate its different functions for therapeutic purposes. In a notable example, IRAP has been targeted with inhibitors that act as cognitive enhancers by reducing brain oxytocin degradation and enhance spine density in primary hippocampal neuron cultures 5-7. More recently, IRAP inhibitors have been developed, targeting its antigenprocessing properties in an effort to regulate adaptive immune responses ${ }^{8-10}$.

We have previously shown that phosphinic pseudotripeptides can act as very potent inhibitors of IRAP 11. Further structure-activity exploration demonstrated that bulky aromatic groups at the P1' side chain of such compounds can result in inhibitors that are highly selective for IRAP ${ }^{8}$. One of those compounds, compound 1 (DG026, ((1R)-1-Amino-3-phenylpropyl)\{2' - 


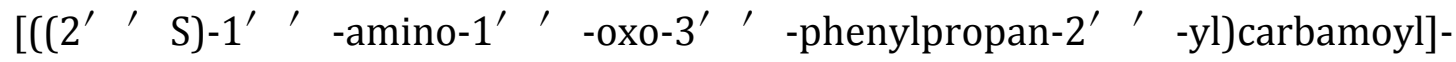
$4^{\prime}, 4^{\prime}$-diphenylbutyl\} phosphinic acid) (Figure 1A) was also shown to be able to selectively down-regulate IRAP-dependent cross-presentation by dendritic cells but leave ERAP1-dependent cross-presentation unaffected ${ }^{8}$. To help understand the structural basis behind this selectivity, we solved the crystal structure of IRAP in complex with $\mathbf{1}$. To our surprise, the structure of IRAP in the complex was significantly different than the ligand-free IRAP or IRAP with a bound peptide ${ }^{12,13}$. This inhibitor-induced conformational change results in a closed conformation in which the internal cavity of the enzyme, that contains the catalytic site, has no access to the external solvent and a new specificity pocket is formed. A key active-site structural motif, the GAMEN loop, is found in a distinct configuration, allowing for additional interactions with the inhibitor and revealing that the active site of IRAP has significant structural plasticity. Our results provide insight on the catalytic mechanism of IRAP and formulate a novel structural framework for understanding its substrate specificity that can be invaluable for the development of potent and selective inhibitors. 


\section{RESULTS}

\section{Compound 1 is a potent and selective inhibitor of IRAP}

A phosphinic pseudotripeptide, $((1 R)-1$-Amino-3-phenylpropyl $)\left(\left(2^{\prime} S\right)-2^{\prime}-\right.$ $\left\{\left[\left(2^{\prime \prime} S\right)-1^{\prime \prime}\right.\right.$-amino-3"'-(1H-indol-3-yl)-1"'-oxopropan-2"'-yl $]$ carbamoyl $\}-4^{\prime}$ methylpentyl) phosphinic acid (DG013), has been described as a very potent inhibitor of all three members of the oxytocinase sub-family of M1 aminopeptidases (ERAP1, ERAP2 and IRAP) but with low selectivity ${ }^{11}$. A later structure-activity study examined the effect of varying the P1' and P2' sidechains on both potency and selectivity ${ }^{8}$. This study resulted in compounds that had enhanced selectivity for IRAP, primarily by incorporating bulky side-chains at position P1'. Accordingly, compound $1^{8}$ carries a diphenyl group at position P1' as well as a non-sterochemically defined chiral center (Figure 1A). The two stereoisomers of $\mathbf{1}$ were separated on reversed-phase HPLC, resulting in compounds $1 \mathrm{~A}$ and $1 \mathrm{~B}$ (Figure 1B). Based on previous work, the first eluted peak is expected to correspond to the $S$ stereochemistry $([\mathrm{R}, \mathrm{S}, \mathrm{S}]$ stereoisomer) and the second to the R stereochemistry ([R,R,S] stereoisomer) ${ }^{11,14}$. This is found to be consistent with our crystallographic analysis for the IRAP-1 complex (see below). In vitro evaluation has suggested that the $\mathbf{1 A}$ stereoisomer is much more potent ${ }^{8}$. 1A was also found to be highly selective for IRAP with IC50 values 10 and 100 fold lower compared to ERAP2 and ERAP1 respectively (Figure 1C). 

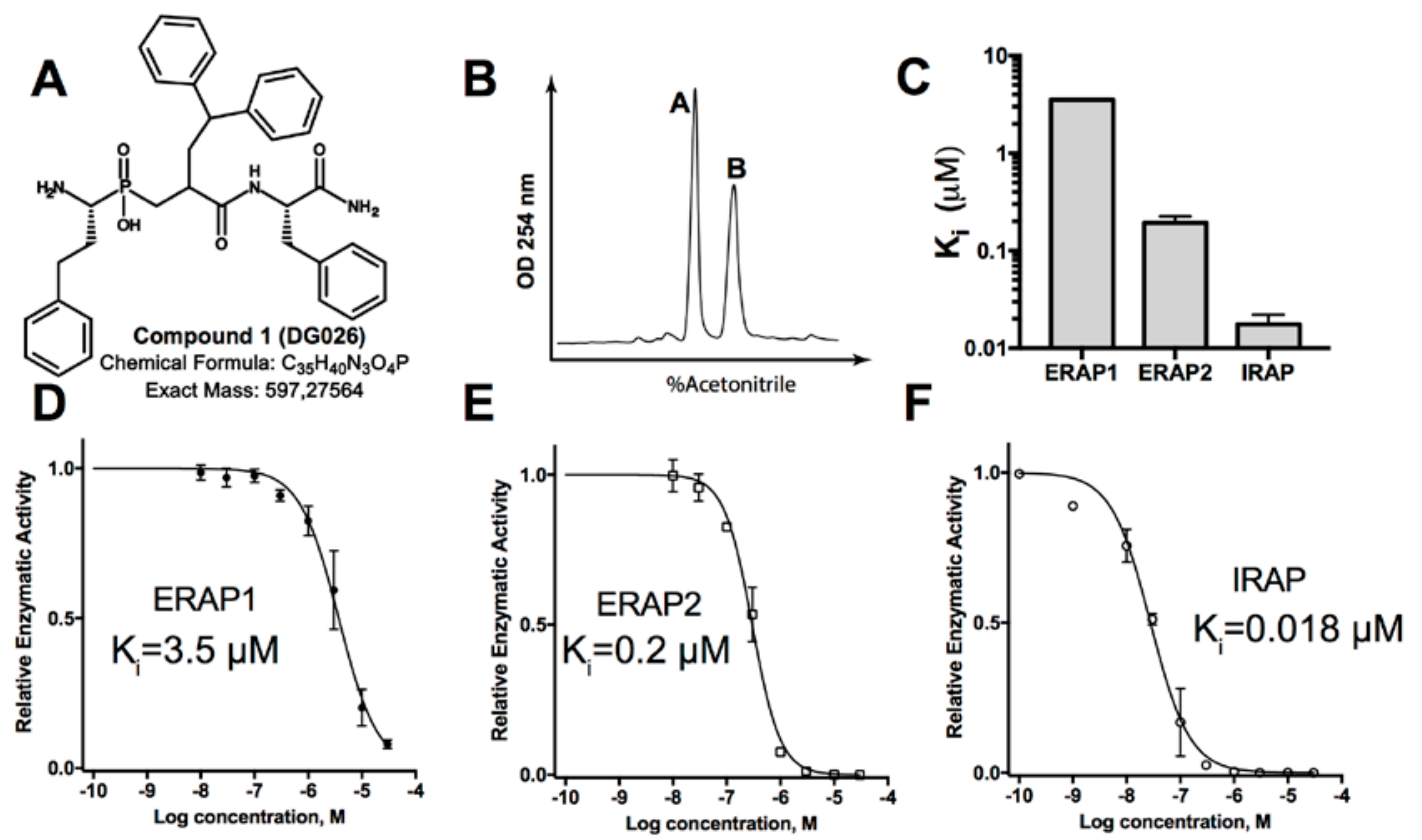

Figure 1: Panel A, chemical structure of inhibitor 1. Panel B, HPLC purification of the two stereoisomers. Panel C, histogram comparing the calculated $\mathrm{K}_{\mathrm{i}}$ values for 1, for ERAP1, ERAP2 and IRAP. Panels D-F, representative inhibition curves of ERAP1, ERAP2 and IRAP by 1.

IRAP undergoes a conformational change upon inhibitor binding.

In our previous study, molecular modeling had suggested that the selectivity of IRAP versus ERAP1 is due to the different configuration of the GAMEN motif in IRAP ${ }^{8,12}$. To test this hypothesis, we generated IRAP crystals according to published conditions 12 and soaked them with 1A. Soaking created significant defects on the crystals that resulted in low-resolution diffraction. As a result, we attempted to co-crystallize pre-formed IRAP-1A complexes as described in the methods section. The best crystal obtained by co-crystallization diffracted to $2.53 \AA$ using synchrotron radiation at the Diamond Light source, the highest resolution reported so far for an IRAP structure. Crystallographic data 
and refinement statistics are shown in Table 1 . The structure was solved by molecular replacement using the highly homologous aminopeptidase ERAP1 (Protein Data Bank entry 2YD0 ${ }^{15}$ ) as a search model.

Surprisingly, the IRAP/1A structure was not identical to either the empty IRAP or IRAP/peptide structure that had been previously solved (PDB codes 5C97, 4P8Q, 4Z7I), which all featured identical domain and active site organization 12,13 . In contrast to the previous structures (henceforth referred to as open conformations), domain IV of IRAP was found to be displaced and was juxtaposed against domains I/II (Figure 2A). This conformational change resulted in full exclusion of the internal cavity from the external solvent (Figure 2B-C). This structure is very similar to the "closed" conformational state of the homologous ERAP1 (PDB code 2YD0) and the only known conformation of ERAP2 (PDB code 3SE6) 15, 16 and is likely to correspond to the active conformation of IRAP 17, 18 . 

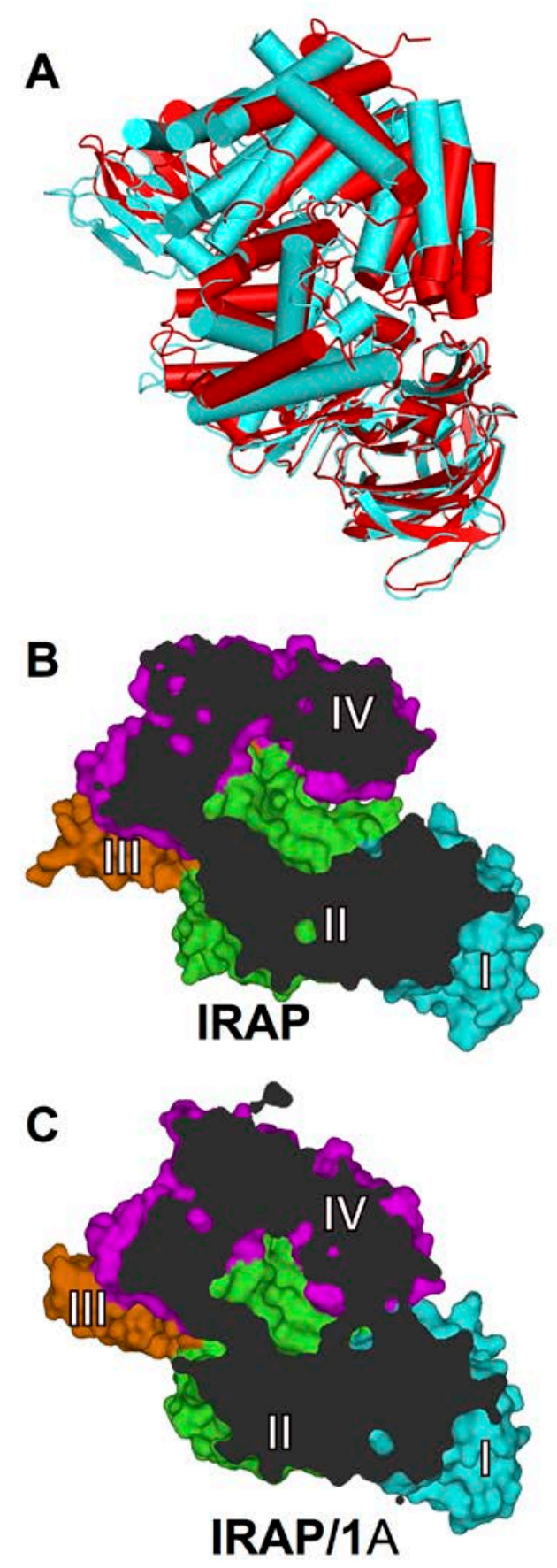

Figure 2: Panel A, cartoon representation of the structure of IRAP in complex with $1 \mathrm{~A}$ (red) aligned with the crystal structure of IRAP in complex with a peptidic substrate analogue (PDB code 4Z7I) or an amino acid (PDB code 5C97) (in cyan). Panels $B-C$, cutaway side-views of the 4Z7I (panel B) compared to IRAP/1A structure (panel C). Domains are color coded and labeled by roman 
numerals. Note the occlusion of the central cavity from the outside solvent in the IRAP/1A structure (panel C).

The bound inhibitor assumes a configuration that is a near-optimal fit to the IRAP active site

Residual electron density in the active site of IRAP was interpreted to belong to compound $\mathbf{1}$. In order to evaluate the stereochemistry of the bound ligand, both compound stereoisomers were used during refinement, but only the $[\mathrm{R}, \mathrm{S}, \mathrm{S}]$ stereoisomer resulted in solutions in which all of the main chain atoms of the inhibitor fit well in the electron density map suggesting that, as predicted, $1 \mathrm{~A}$ is the $[R, S, S]$ stereoisomer (Figure $3 \mathrm{~A}$ ). The generated ligand model lies in a very snug fit inside the cavity formed around the active site, in the space between domains I/II and the juxtaposed domain IV (Figure 3B). Space for additional atoms is only available at the C-terminus of the pseudopeptide, a configuration that would allow the accommodation of longer peptidic substrates in this conformation of IRAP. Several interactions with main chain and side-chains of residues of IRAP were found to stabilize the inhibitor (Figure 4 and Figure S1). The majority of these interactions were identical to the interactions described for a 10 mer peptide 12 with some notable differences: i) the interaction of Tyr961 with the N-terminal phenyl group of the inhibitor, ii) the interaction of Phe550 with one of the phenyl rings of the 2-diphenyl ethyl moiety of the inhibitor as well as the hydrophobic interaction of Leu457 and Ile461 with the other phenyl ring and iii) hydrogen bonding between the carbonyl group of the inhibitor amide bond with the main chain amide of Gly428 as well as 
hydrophobic interactions of carbon atoms of the inhibitor with Ala427 and Ala429.

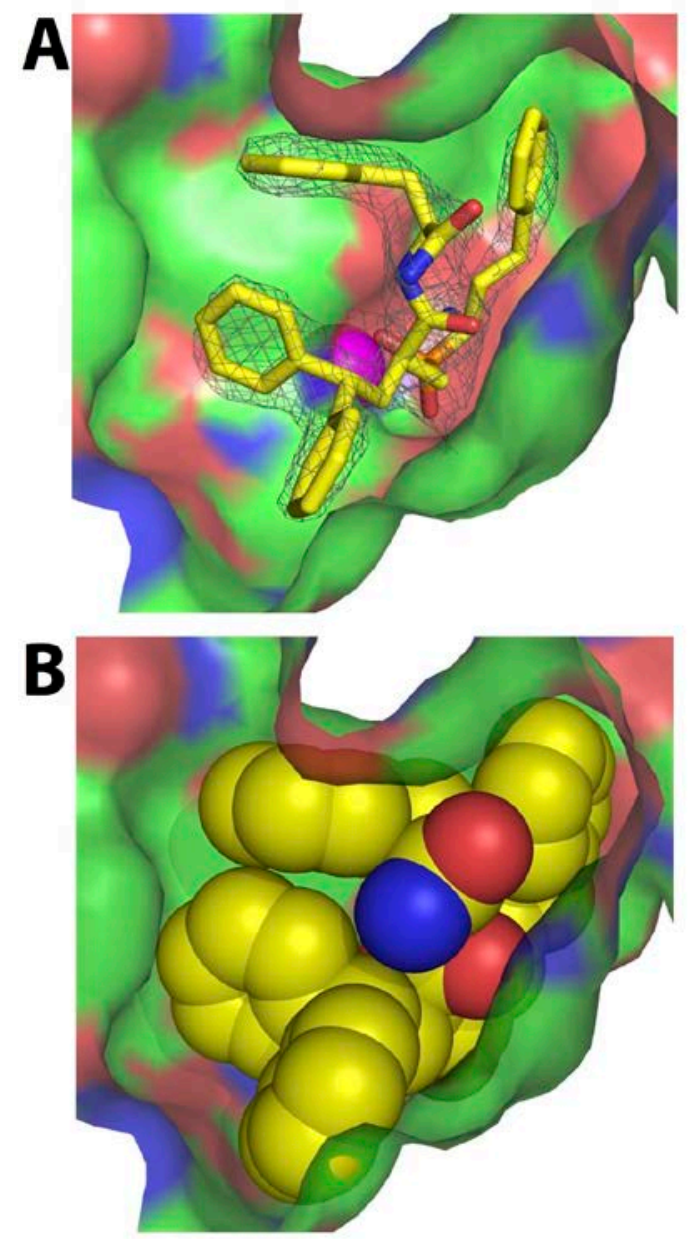

Figure 3: Panel $A$, atomic model of the $1 \mathrm{~A}$ compound ([R,S,S] isomer) in the active site of IRAP. The inhibitor is shown in stick representation (carbon in yellow, oxygen in red, nitrogen in blue) and the IRAP active site in surface representation (carbon in green, nitrogens in blue, oxygens in red). The $2\left|F_{0}\right|-\left|F_{c}\right|$ post-refinement electron density map is shown as a blue mesh contoured at 2.0 $\sigma$. Helices 9 and 10 of domain IV are shown in orange. Panel B, same as in A, but the inhibitor atoms are shown as spheres to highlight the shape complementarity with the IRAP active site. 


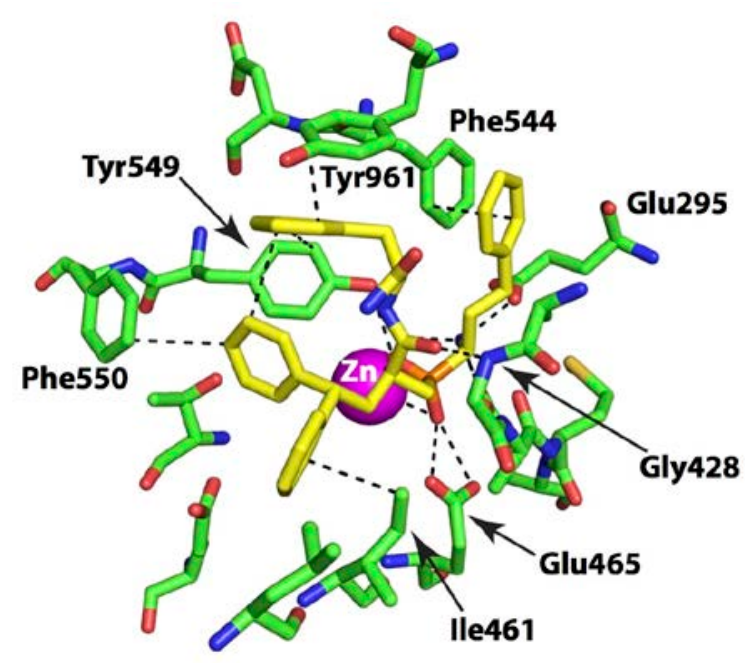

Figure 4: Network of interactions that stabilize compound $\mathbf{1 A}$ in the active site of ERAP1. The inhibitor is shown in yellow and IRAP side chains atoms within 4 $\AA$ of the inhibitor are shown in green. Dotted lines indicate particular side chain interactions between 3-4 $\AA$. The catalytic $\mathrm{Zn}(\mathrm{II})$ atom is shown as a magenta sphere and dotted lines indicate enzyme-ligand interactions.

Ligand binding alters the structural configuration of key residues in the active site

Comparison between the open conformations of IRAP and the closed IRAP/1A structure revealed key structural reorganization of active site residues that mediate interactions with the inhibitor, which may therefore be promoted by inhibitor binding. Notably, the orientation of the GAMEN loop, a key structural motif in M1 aminopeptidases is significantly altered. Specifically, Phe425, Glu426 and Ala427 are in completely different orientations (Figure 5A). Compared to the open IRAP conformation, the reorientation of the GAMEN loop is required in the closed structure due to steric hindrance from the approaching helices $\alpha 9$ and $\alpha$ 10 of domain IV (Figure 5A). This reconfiguration results in the GAMEN loop abutting onto the bound inhibitor, making several van der Waals and hydrogen 
bonding interactions (Figure 5B). It should be noted that in a previously determined open conformation of IRAP in complex with a 10mer substrate analogue and although the active site was occupied, the GAMEN loop was oriented away from the bound ligand, creating empty space that was hypothesized to be available for binding of cyclic peptides. Comparison of these two IRAP/ligand-bound structures suggests that the GAMEN loop in IRAP has significant structural plasticity, an observation that, to our knowledge, is novel for this family of aminopeptidases.

Another significant change in active-site residues is the translocation of Tyr961 (Figure 5B). Tyr961 is located on domain IV of the enzyme and in the open conformation is located $6.2 \AA$ away from the N-terminal phenyl group of the inhibitor. In the closed conformation, Tyr961 makes $\pi$-stacking interactions with this phenyl group, which should enhance inhibitor affinity and may promote the conformational change of IRAP.
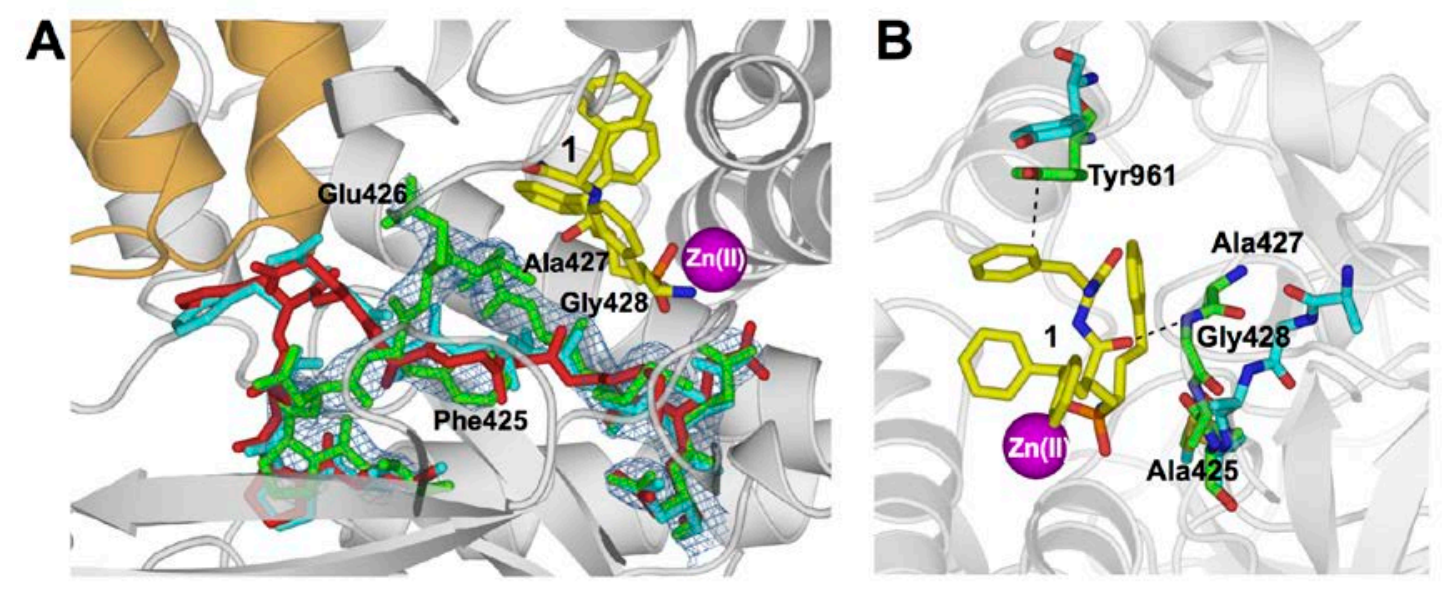

Figure 5: Panel A, configuration of the GAMEN loop in the IRAP/1A complex (green) compared to IRAP structures with PDB codes 4Z7I (cyan) and 5C97 (red). Compound $\mathbf{1 A}$ is indicated with yellow sticks and the active-site $\mathrm{Zn}$ (II) atom in shown as a magenta sphere. The $2\left|\mathrm{~F}_{\mathrm{o}}\right|-\left|\mathrm{F}_{\mathrm{c}}\right|$ electron density map for the 
GAMEN loop is shown as a blue mesh, contoured at 2.0\%. Domain IV helices 9 and 10 that pack against the GAMEN loop are shown in orange. Panel B, schematic representation of the structural changes that generate additional interactions with the inhibitor in the active site. Residues from the open conformation of IRAP (PDB code 4Z7I) are shown in cyan sticks and residues from the closed conformation (IRAP/1A) are shown in green sticks. Dotted lines represent distances below $4 \AA$.

To better understand the potential effect of the inhibitor in mediating the transition from the "open" state of IRAP observed in its complexes with substrates ${ }^{13}$ and phosphinic peptides 12 towards the closed state described here, we employed molecular dynamics (MD) simulations using the X-ray structure of IRAP/1A and a model of IRAP/1A in the open state as described in the methods section. Our simulations at the sub-microsecond timescale using the latest AMBER force field showed that both configurations are relatively stable in that timescale, with only minimal deviations from the X-ray structures (Supporting Figure S2). Specifically, the inter-domain angle (used as a metric of domain closure) fluctuated around the values observed in the X-ray structures of IRAP, around $55 \mathrm{deg}$ in the open state and $50 \mathrm{deg}$ in the closed state. These data indicate that the open-to-closed transition possibly occurs in a slower timescale that is not accessible by these simulations. Therefore, we used targeted MD ${ }^{19}$ to monitor the effect of the conformational shift of residues 423-432 (the GAMENmotif including 5 preceding residues) starting from the open IRAP/1A model towards the configuration observed in the closed state (Supporting Figures S3, S4). During this transition, we observed a decrease in the root-mean square deviation of the whole complex with respect to the closed IRAP, a decrease of the 
interdomain angle towards $53 \mathrm{deg}$ and the formation of two stable hydrogen bonds between the amide carbonyl oxygen of the inhibitor and the amine $\mathrm{NH}$ groups of Gly428 and Ala429 (Supporting Figures S5, S6). After imposing the transition in these 10 residues only (Supporting Movie S2), we monitored the conformational changes of the enzyme through unrestrainted MD simulations. Under these conditions, the overall structure of IRAP readily converged - within $0.2 \mu$ s of the simulation - to the closed structure. This finding suggests that the reconfiguration of the GAMEN loop can act as a conformational trigger for a broader IRAP conformational change and supports the notion that the transition from the open to closed conformation can be mediated by interactions between the inhibitor and the GAMEN loop (Supporting Figures S5, S6).

\section{Structural determinants for inhibitor selectivity}

The strong selectivity of $1 \mathrm{~A}$ for IRAP must rely on structural elements that are unique to the enzyme. However, being a mechanism-based inhibitor, significant affinity is generated by structural elements common to all homologous enzymes: i) the active site $\mathrm{Zn}(\mathrm{II})$ atom, ii) the catalytic Tyr549 and Glu465, iii) N-terminus recognition by Glu295, Glu431 and Glu487 and iv) the S1 specificity residue Phe544. We previously attempted to explain the selectivity of 1A by computational docking calculations using the open conformation of IRAP and proposed a binding configuration in which the P1' side-chain abuts on the GAMEN loop and as a result the selectivity for IRAP is driven by the different orientation of this loop ${ }^{8}$. The structure presented here however does not validate that hypothesis since the GAMEN loop is now reoriented and is in a similar conformation as the GAMEN loop in ERAP2 and ERAP1. 
To understand the observed selectivity we superimposed the closed conformations of ERAP1, ERAP2 and IRAP and analyzed the atomic interactions of the inhibitor with all non-conserved residues (Figure 6). Notably, ERAP1 has a serine residue in the equivalent position to Tyr961, which should be unable to provide favorable interactions with the inhibitor. Similarly, the ERAP1 residue Ser316 is in the homologous position to IRAP's Ala427, which would result in steric hindrance with the inhibitor's P1 side-chain as well as unfavorable interactions between the Ser316 hydroxyl group and the P1 aromatic ring. Finally, the ERAP1 residue Lys380 would be located in a very close proximity to one of the phenyl groups of the 2-diphenyl ethyl P1' side-chain generating steric hindrance. Similar unfavorable interactions would be expected between Asp435 and the phenyl P1 side-chain. Overall, the Phe550-to-basic, Thr491-to-acidic and Ala427-to-polar substitutions create a more polar/charged environment that is less optimal for a hydrophobic ligand. In summary, at least 4 unfavorable interactions between ERAP1 and this inhibitor should be sufficient to explain the lower affinity against this enzyme. 


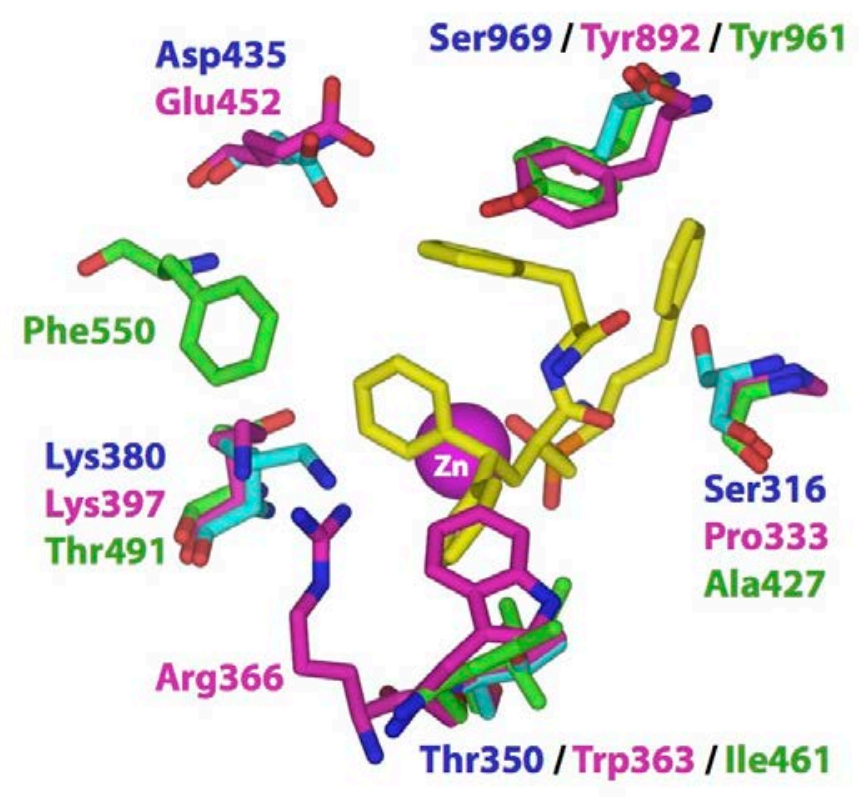

Figure 6: The inhibitor 1A (yellow sticks) is shown in the active site of IRAP. Non-conserved residues in the active site between ERAP1 (cyan), ERAP2 (magenta) and IRAP (green) are also indicated.

The reduced affinity of the inhibitor for ERAP2 may also be understood in the context of unfavorable interactions: i) steric hindrance from Trp363, ii) the position of Arg366 and Lys397, iii) unfavorable position of Glu452 (Figure 6). Interestingly there are fewer unfavorable interactions between ERAP2 and 1A than between ERAP1 and 1A consistent with the intermediate affinity for ERAP2. Overall, structural analysis strongly suggests that the high affinity and selectivity of this compound for IRAP is a direct result of the near-optimal shape complementarity and atomic interactions with a network of residues in the active site of the enzyme. It is conceivable that this optimized network is sufficient to drive the conformational change of IRAP from the open to the closed conformation. It is worthwhile to note that the selectivity of $1 \mathrm{~A}$ can be more 
readily understood with regard to the closed conformation of IRAP, suggesting that this conformation may be very useful for future rational design inhibitor efforts.

To further investigate the importance of the two conformations of IRAP in driving inhibitor selectivity we used computational modeling to dock a benzopyran derivative, compound 2, (HFI-437, Ethyl 2-acetamido-7-hydroxy-4(3-quinolinyl)-4H-chromene-3-carboxylate)) previously shown to be a potent IRAP inhibitor and to display cognitive enhancing activity in mouse models (Figure 7A) 13, 20. Using molecular docking calculations, Hermans et al. proposed that compound $\mathbf{2}$ as well as other similar benzopyran derivatives, bind IRAP in an almost identical fashion, with the chromenol moiety packed against the GAMEN loop and its hydroxyl group interacting with the catalytic zinc (see Figure 7A for a similar pose $)^{13}$. According to that binding mode, the pyridinyl or quinolinyl substituents of the benzopyran derivatives are stacked against Phe544, which can help explain the sensitivity of these compounds to mutations at Phe544 ${ }^{21}$. However, such a bound pose for 2 (Figure 7B) is not possible in the closed conformation of IRAP due to steric clashes with GAMEN-motif residues. Instead, docking calculations using the closed IRAP conformation predict preferred configurations in which the chromenol group lies inside the S1 specificity pocket and its aromatic substituent is stacked between the catalytic Tyr549 and Tyr809 from domain IV (see for example the (R)-enantiomer of 2 in Figure 7C). In such a configuration, i) the carbonyl group of the 2-acetamide moiety interacts with $\mathrm{Zn}(\mathrm{II})$, ii) its amide $\mathrm{N}-\mathrm{H}$ forms a hydrogen bond with the carbonyl group of Ala429, iii) the 3-ethylcarboxylate moiety is hydrogen bonded with the backbone N-H groups of Gly428 and Ala429 in the GAMEN loop, and iv) 
the 7-OH group of the inhibitor exhibits a hydrogen bond with the backbone carbonyl group of Glu295. This alternative configuration is mediated by the more closed conformation of the GAMEN motif and the closure of domain IV that results in the approaching of the two tyrosine residues, Tyr549 and Tyr961. The sensitivity of 2 to mutation of the Phe 544 can be also explained by the proposed pose of Figure 7B: destabilization of the stacking interaction between the chromenol moiety and Phe544Ala mutant would result in destabilization of the stacking interaction between the 4-position quinolinyl or the pyridinyl substituent between the two conserved Tyr549/961 residues, with the latter been more pronounced given the lower aromatic ring overlap. The possibility for such extensive differences in inhibitor binding modes in the two available IRAP structures, necessitates determining co-crystal structures of IRAP with these and other inhibitors along with more extensive molecular docking / molecular dynamics studies using both IRAP conformations, for the design and discovery of next-generation of potent and selective IRAP inhibitors. 
A<smiles>CCOC(=O)C1=C(NC(C)=O)Oc2cc(O)ccc2C1c1cnc2ccccc2c1</smiles>

Compound 2 (HFI 437)

B

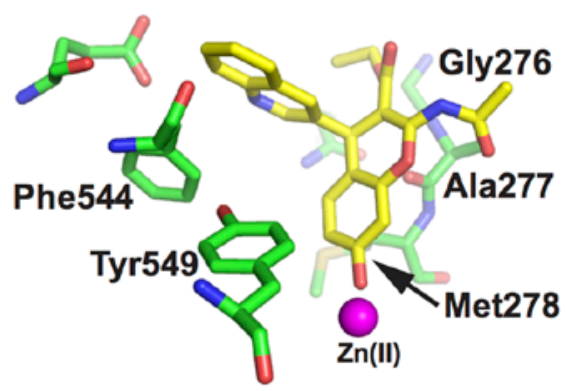

C

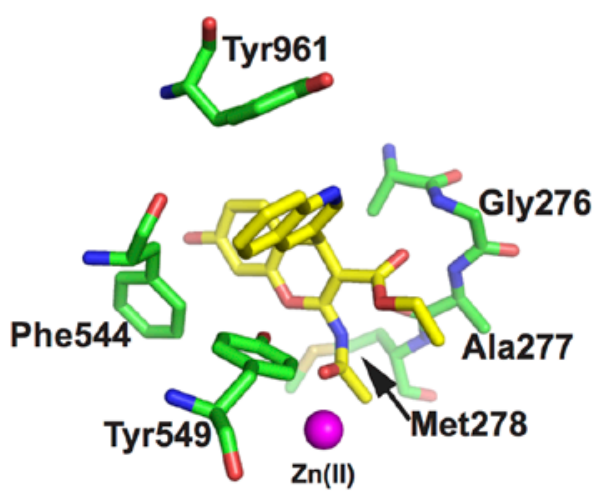

Figure 7: Panel A, chemical structure of compound 2. Panels B-C, docked conformations of compound 2 (yellow sticks) in the active site of the open conformation of IRAP (panel B, PDB: 4PJ6) and the closed conformation of IRAP (panel C, PDB: 5MJ6). Key interacting residues from the active site of IRAP are shown in green sticks. Note that the pose shown in panel B would generate steric clashes with the GAMEN loop in the closed conformation of IRAP shown in panel C. 


\section{DISCUSSION AND CONCLUSIONS}

Although three crystal structures of IRAP have been described before, one with an empty active site and two with bound ligands (an amino acid or a 10mer peptide analogue), no significant conformational change was observed between them 12, 13. It was therefore surprising to discover such a significant conformational change upon binding of the inhibitor 1A. Furthermore, this conformational change appears to have been induced by inhibitor binding since in soaking experiments the inhibitor would damage a preformed crystal of IRAP in the open conformation. It can, therefore, be concluded that the nature of the interaction of the ligand with IRAP is the primary determinant that induced the conformational change. Indeed, the $\pi$-stacking interaction between the domain IV residue Tyr961 and the phenyl group of the P2' side-chain of the inhibitor may have been the catalytic force. Aryl-aryl stacking interaction energy can vary widely depending on ring nature and exact orientation but is usually within the 1-3 kcal/mol range ${ }^{22}$. Computational analysis of the homologous ERAP1 however has suggested that similar conformational changes as the one reported here for IRAP may take place on a highly rugged energy landscape, although the energy barriers associated with the inter-conversion between the open-to-closed states are relatively low ${ }^{23}$. It is therefore possible that key atomic interactions between a ligand and IRAP can promote conformational closing. Indeed, a similar conformational change has been proposed for ERAP1 to be a key component of its catalytic cycle ${ }^{18}$. Given the structural and functional similarities between the two enzymes, it is therefore reasonable to postulate that the conformational change from open to closed structures is also a key component of the IRAP 
catalytic cycle. The open conformation is responsible for initial substrate capture, which can induce further closing that enhances interactions and facilitates catalysis. In this context, it may be considered surprising that the only known co-crystal structure of IRAP with a peptide, is in an open conformation ${ }^{12}$. It should be noted however, that even in the open conformation, IRAP is significantly more closed compared to the open conformation of ERAP1 18 and that further closing appears to be hindered due to the abutting of the $\mathrm{C}$-terminal moiety of the peptide in the limited space between domains II and IV, and thus may represent the limit on how much IRAP can close with that particular peptide bound.

Our analysis suggests that the GAMEN loop rearrangement is linked to the overall conformational change in IRAP and in particular to the approach of helices $\alpha 9$ and $\alpha 10$ of domain IV towards the bound inhibitor. This is further supported by our MD calculations that suggest that the reconfiguration of the GAMEN loop can act as a conformational trigger in mediating complete closure of IRAP. A visualization of this conformational change generated using the elastic network model server ${ }^{24}$ can be seen in the Supporting Movie M1, whereas an animation of the GAMEN loop conformational transition during the targeted MD simulation is shown in Supporting Movie M2. Interestingly, although the highly homologous ERAP1 undergoes a similar conformational change, no significant rearrangements of its GAMEN motif have been observed and even in the open state of ERAP1 the GAMEN motif is in a similar orientation as in the closed state 18. Overall, comparing the GAMEN motif conformation from all known structures of ERAP1, ERAP2 and IRAP suggests that it is the orientation of the GAMEN loop in the open conformation of IRAP that is unique $12,13,15,16,18$. This may therefore 
be a unique property of IRAP that is closely related to its multifaceted biological function since a highly plastic GAMEN motif can allow the accommodation of a wide range of substrates, including cyclic peptides ${ }^{13}$. It is also possible that the conformational plasticity of the GAMEN motif can allow for the efficient processing of a greater variety of antigenic peptide precursors by IRAP 25 . Indeed, although ERAP1 cooperates with ERAP2 in generating antigenic peptides 26, IRAP has to perform a similar function by itself 27 and possibly for this reason also combines the N-terminal specificity of both ERAP1 and ERAP2 28.

The conformational plasticity of the active site of IRAP would be expected to have repercussions on the development of potent and selective IRAP inhibitors. Accordingly, although several different classes of compounds have been described as potent inhibitors of IRAP 5, 6,8,9,20,29, clear structure-activity relationships have been elusive and may be complicated by the intrinsic plasticity of the active site. This is further supported by our docking calculations using 2 to both known conformations of IRAP which suggest two plausible but completely different binding configurations of the inhibitor depending on the IRAP conformation. Since the active site has no direct access to the solvent in the closed conformation, the initial encounter complex with any substrate or inhibitor would unavoidably have to be with an open conformation. As a result, a successful inhibitor has to be able to bind with high affinity to both conformations or possibly induce conformational closing, as it appears to be in the case of inhibitor $\mathbf{1 A}$. On the other hand, the structural adaptability of the IRAP active site generates a major hurdle to overcome in designing inhibitors for homologous enzymes that are inactive for IRAP. This may as well be the case for developing inhibitors for ERAP1 and ERAP2, which have recently emerged as 
tractable targets for cancer immunotherapy ${ }^{30}$. Indeed, two recently published structure-activity studies on these three enzymes that focused on rationally designed active-site-targeting compounds, revealed an exquisite difficulty in generating ERAP1 inhibitors that are inactive against IRAP but not vice-versa ${ }^{8,9}$. Generating ERAP1-selective inhibitors that do not target IRAP may be highly pharmacologically relevant given the multitude and complexity of biological functions that IRAP is involved in, resulting in possibly serious side-effects from off-target IRAP inhibition. As a result, future structure-based inhibitor design efforts need to take into account the conformational plasticity of IRAP, especially with respect to the $\mathrm{S} 1$ pocket and the GAMEN loop region.

In summary, we describe a novel conformation of Insulin-Regulated Aminopeptidase, an enzyme with multiple important biological roles including antigen processing. This conformation is brought about by binding of a potent and selective inhibitor and may constitute the active conformation of the enzyme. The altered configuration of the active site GAMEN loop reveals a significant plasticity of the active site of IRAP that has not been previously realized, may relate to the enzyme's diverse biological functions and needs to be taken into account when designing small-molecule inhibitors that select for or against IRAP. 


\section{EXPERIMENTAL SECTION}

\section{Protein expression and purification}

Expression and purification of the soluble domain of recombinant IRAP was performed as described previously ${ }^{12}$. Briefly, IRAP was isolated after secretion from stably-transfected HEK $293 \mathrm{~S}$ GnTI(-) cells and purified by affinity chromatography (anti-rho1D4 tag Ab, elution with rho1D4 peptide) and sizeexclusion chromatography (Superdex 200 16/60 column; GE Healthcare) in 10 $\mathrm{mM}$ Hepes (pH 7.5) and $150 \mathrm{mM} \mathrm{NaCl}$.

\section{Inhibitor synthesis and purification}

The synthesis of compound $\mathbf{1}$ has been described before (as compound $22 \mathrm{~b})^{8}$. Isolation of the $\mathbf{1 A}$ isomer was performed by reverse-phase HPLC (Merck Chromolith C-18 column) using a 0.05\% TFA-acetonitrile gradient (5-40\%). Purity was determined by analytical HPLC to be $>95 \%$.

\section{Enzymatic assays}

The aminopeptidase activity of recombinant IRAP was measured by following the change in fluorescent signal produced upon digestion of the substrate L-leucine 7-amido-4-methyl coumarin (Sigma-Aldrich). The fluorescence was measured at $460 \mathrm{~nm}$, whereas the excitation was set at $380 \mathrm{~nm}$. Measurements were performed on a TECAN infinite M200 microplate fluorescence reader as previously described ${ }^{31}$. Evaluation of the inhibitory potency of the compounds was carried out using the same fluorimetric assay as previously described ${ }^{11}$. Calculation of the inhibitor $\mathrm{K}_{\mathrm{i}}$ values for each enzyme 
was performed as described ${ }^{32}$ using previously calculated $\mathrm{K}_{\mathrm{M}}$ values for each substrate ${ }^{28}$.

\section{Crystallization and collection of diffraction data}

Crystallization trials were performed by sitting drop vapour diffusion in 96-well plates (Greiner Bio-One, Stonehouse, U.K.), using a Cartesian Technologies Microsys MIC4000 liquid-handling robot at $21^{\circ} \mathrm{C}$. Crystallization droplets were imaged at regular intervals with an RI1000 imaging system (Formulatrix, Bedford, USA). Purified IRAP at a concentration of $7.5 \mathrm{mg} / \mathrm{mL}$ in $150 \mathrm{mM} \mathrm{NaCl}$ and $10 \mathrm{mM}$ Hepes buffer $(\mathrm{pH}$ 7.5) was screened for cocrystallization with 1A against serial dilutions of Morpheus Screen ${ }^{33}$ conditions (Molecular Dimensions Ltd.) under which native IRAP crystals had been previously obtained. Crystals of IRAP in complex with 1A were obtained by adding a 5-fold molar excess of the ligand to the concentrated protein and incubating for $1 \mathrm{~h}$ at room temperature. For soaking experiments, $1 \mathrm{~A}$ dissolved in water at $2 \mathrm{mM}$ was mixed at a 1:10 ratio with the reservoir conditions (B9 condition of the Morpheus screen, Molecular Dimensions Ltd.), then diluted 1:1 with the drop containing the crystal and incubated for $5 \mathrm{~h}$ before freezing. Data were collected and analyzed as described above. The best data for the IRAP/1A complex were collected from a crystal obtained from the following reservoir condition: 18.8\% (w/v) PEG of mean MW 20000, 37.6\% (v/v) PEG monomethyl ether of mean MW 500, $50.2 \mathrm{mM}$ Bicine, $43.8 \mathrm{mM}$ Trizma base $(\mathrm{pH}$ of buffer mixture: 8.5 ) and $0.282 \mathrm{M}$ each of the following halogen salts: Sodium fluoride, Sodium bromide and Sodium iodide. The crystal belonged to space group P212121 with $\mathrm{a}=112.24 \AA, \mathrm{b}=143.17 \AA$ and $\mathrm{c}=148.99 \AA$. The resulting dataset 
(collected at $100 \mathrm{~K}$ ) displayed useful data to $2.53 \AA$ A. $5 \%$ of the reflections were flagged for Rfree calculations. Data were collected at beamline I03 at the Diamond Light Source UK, equipped with a Pilatus3 6M pixel detector and were merged and scaled using $x i a 2^{34}$.

The structure was solved by molecular replacement with Phaser ${ }^{35}$, using the closed conformation of the highly homologous aminopeptidase ERAP1 (Protein Data Bank [PDB] entry 2YD015) as a search model. Two protein molecules were found in the asymmetric unit. Refinement was performed using programs Refmac ${ }^{36}$ at the initial stages and Phenix.refine ${ }^{37}$ at the later stages. Coot and JLigand was used for building the protein and the ligand ${ }^{38}$. The density of the ligand was fully apparent in both chains. The refinement converged to $\mathrm{R}$ and Rfree of 17.33 and $22.90 \%$, respectively. In chain A, no density was visible before residue 157 , and between residues $640-648$. In chain $B$, no density was visible before residue 160 and between residues 639-648. We also included in the model 44 molecules of N-acetyl-D-glucosamine, 9 of $\beta$-D-mannose, 6 of $\alpha$-Dmannose, 21 bromide ions and 240 water molecules.

\section{Computational methods}

Preparation of the simulation systems: Two simulation systems were prepared on the basis of the X-ray crystal structures of IRAP complex with a lysine substrate (PDB ID: 4PJ6) ${ }^{13}$ in the "open" state, and the current structure of IRAP-1A complex in the "closed" state. To examine the effect of the inhibitor in the open structure of IRAP, the Lys substrate was substituted by $\mathbf{1 A}$ after superimposing the two X-ray structures with respect to domain I and II residues. Only the protein, ligand and zinc atoms of chains A were used, whereas the 
alternative location B atoms were discarded. The missing loop residues were added using MODELLER (v9.10) ${ }^{39}$ and then hydrogen atoms were added at physiological $\mathrm{pH}$ (7.4) using the $\mathrm{H}++$ server. $^{40}$ In particular, histidine residues 255, 570, 579 and 830 were set as positively charged, the zinc-bound His464/468 and histidines 528, 653979 as protonated at $\mathrm{N}^{\delta 1}$, whereas all other were protonated at $\mathrm{N}^{\varepsilon 2}$. A disulfide bond was created between Cys828 and Cys835, zinc was bonded to His464/His468/Glu487, and the ligand was bonded to zinc via both phosphinic oxygen atoms using the LEaP module of AMBER v16. ${ }^{41}$ The ff14SB parameters were applied to protein atoms ${ }^{19}$, GAFF force-field parameters with AM1-BCC charges were calculated for 1A using ANTECHAMBER 42, 43, and parameters for the zinc-binding group were taken from ref. ${ }^{44}$ The systems were solvated in truncated octahedral boxes comprising TIP3P ${ }^{45}$ water molecules with a minimum distance of $10 \AA$ between protein and the edge of the periodic box. Charge neutralization and an ionic strength of $\sim 0.15 \mathrm{M}$ were achieved by adding $100 \mathrm{Na}^{+}$and $91 \mathrm{Cl}^{-}$ions.

Molecular docking calculations: The initial conformation of the small molecule inhibitor $2^{20,29}$ was prepared in both enantiomeric forms from SMILES representations using VIDA. ${ }^{46}$ Proteins and ligands were treated with non-polar hydrogen atoms only and Gasteiger charges were applied using AutoDockTools (v1.5.6). The search space was defined by a grid box centered next to the catalytic zinc and comprised $66 \times 66 \times 66$ grid points of $0.375 \AA$ spacing. For each ligand, 100 docking rounds were calculated using the Lamarckian genetic algorithm with default parameters in AutoDock (v4.2.6). ${ }^{47,} 48$ The maximum number of energy evaluations was set to $10 \times 10^{6}$ and the docked conformations were clustered using a tolerance of $2.0 \AA$. 
Conventional molecular dynamics (cMD) simulations were performed with the GPU-version of PMEMD program using periodic boundary conditions ${ }^{49}$. A time step of 4.0 fs was used after repartitioning the mass of heavy atoms into the bonded hydrogen atoms according to the HMR scheme implemented in ParmEd (v2.6) ${ }^{50}$. The temperature was controlled using a Langevin thermostat with a collision frequency of $5.0 \mathrm{ps}^{-1,51}$ and the pressure was regulated at 1 bar using the Berendsen weak-coupling algorithm with a relaxation time of $5.0 \mathrm{ps}^{52}$. Electrostatic interactions were evaluated by means of the Particle Mesh Ewald method ${ }^{53}$ with a real space cutoff of $8.0 \AA$ and a direct sum tolerance of $10^{-6}$. The center-of-mass of the solute was reset to zero every 1,000 steps and the reciprocal sum was calculated every single step. Each system was first energy minimized to remove any close contacts and then harmonic positional restraints (force constant of $50 \mathrm{Kcal} \cdot \mathrm{mol}^{-1} \cdot \AA^{-2}$ ) were applied to the protein backbone atoms. The temperature was increased from $10 \mathrm{~K}$ to $300 \mathrm{~K}$ as a linear function of time over the course of 100 ps simulation under constant volume (NVT ensemble). The restraints were gradually removed over 9 rounds of 100 ps in the isothermal-isobaric (NPT) ensemble, and then an additional unrestraint simulation was carried out for 9 ns under constant pressure of 1 bar and temperature of $300 \mathrm{~K}$. Production runs were performed for 200-400 ns in the NPT ensemble under the same conditions, employing the Monte Carlo barostat introduced in AMBER 16.

Targeted molecular dynamics were performed starting from the equilibrated structure of open IRAP-1A model. The closed IRAP-1A structure presented here was used as the target structure for residues 423-432, and residues 388-473 were used for the root mean square deviation (RMSD) fitting. 
The target mass-weighted RMSD for the non-hydrogen atoms of residues 423432 was decreased to zero within 1 ns of targeted MD simulation in the NVT using the SANDER module. A force constant of $2 \mathrm{Kcal} \cdot \mathrm{mol}^{-1} \cdot \AA^{-2}$ was employed for the targeted MD restraint energy term.

Trajectory processing and analysis was performed with the CPPTRAJ module of AmberTools (v15) ${ }^{54}$ and visual inspection of the trajectories and rendering of the figures was performed with VMD (v1.9) ${ }^{55}$. Calculations were performed on an Intel Xeon server equipped with NVIDIA GTX780 GPUs with CUDA 5.0.

\section{SUPPORTING INFORMATION}

Ligplot diagram showing the interaction between inhibitor and residues of IRAP; Movie depicting the IRAP conformational change based on analysis according to the Elastic Network Model server; Plots depicting parameter changes during molecular dynamics simulations; Schematic representation of molecular modeling strategy employed to study the effect of the GAMEN-motif reconfiguration; Close-up view of the conformational changes imposed at residues $423-432$ in the open structure of IRAP in complex with the inhibitor; Animation showing the conformational change of the GAMEN-motif and five preceding residues during the course of the targeted MD simulation; Molecular Formula Strings File containing the structure of the inhibitor.

\section{ACKNOWLEDGMENTS}

This research was financed by the European Union (European Social Fund) and Greek national funds through the Operational Program "Education 
and Lifelong Learning" of the National Strategic Reference Framework: Research Funding Program of the General Secretariat for Research \& Technology (grant no. ERC-14 to EfS). Funding was also provided by the Harry J. Lloyd Charitable trust (to EfS). The research leading to these results has also received funding from the European Community's Seventh Framework Programme (FP7/20072013) under BioStruct-X (grant agreement $\mathrm{N}^{\circ} 283570$ ). K.H. is supported by MRC Grant MR/N00065X/1. The Wellcome Trust Centre for Human Genetics is supported by the Wellcome Trust (grant 090532/Z/09/Z). We thank Diamond Light Source for beamtime (proposal MX10627) and the staff of beamline I03 for assistance with data collection.

\section{ACCESSION CODES}

Atomic coordinates and structure factors have been deposited in the Protein Data Bank as entry 5MJ6. The authors will release the atomic coordinates and experimental data upon article publication.

\section{CORRESPONDING AUTHOR}

Address correspondence to: Dr. Efstratios Stratikos, National Centre for Scientific

Research Demokritos, Agia Paraskevi, Greece, 15341. Email: stratikos@rrp.demokritos.gr or stratikos@gmail.com. Telephone: +30-2106503918, Fax: +30-210-6543526.

\section{AUTHOR CONTRIBUTIONS}

AM produced recombinant protein with help from YZ, performed crystallization and solved the crystal structure. EmS performed crystallization 
trials, processed data and helped solve the crystal structure with assistance from PG. KH helped with crystallization and performed diffraction experiments and data processing. PK and DG synthesized and purified the inhibitor. AP performed the computational modeling and interpretation. ES conceived and supervised the project, helped solve the crystal structure, interpreted data and wrote the paper with input from all authors.

\section{ABBREVIATIONS USED}

IRAP, Insulin Regulated Aminopeptidase; ERAP1, Endoplasmic Reticulum Aminopeptidase 1; ERAP2, Endoplasmic Reticulum Aminopeptidase 2.

\section{REFERENCES}

1. Hattori, A.; Tsujimoto, M. Endoplasmic reticulum aminopeptidases: biochemistry, physiology and pathology. J. Biochem. 2013, 154, 219-228.

2. Ye, S.; Chai, S. Y.; Lew, R. A.; Ascher, D. B.; Morton, C. J.; Parker, M. W.; Albiston, A. L. Identification of modulating residues defining the catalytic cleft of insulin-regulated aminopeptidase. Biochem. Cell Biol. 2008, 86, 251-261.

3. Evnouchidou, I.; Papakyriakou, A.; Stratikos, E. A New Role for Zn(II) Aminopeptidases: Antigenic peptide generation and destruction. Curr. Pharm. Des. 2009, 15, 3656-3670.

4. Saveanu, L.; van Endert, P. The role of insulin-regulated aminopeptidase in MHC class I antigen presentation. Front. Immunol. 2012, 3, 57.

5. Albiston, A. L.; Diwakarla, S.; Fernando, R. N.; Mountford, S. J.; Yeatman, H. R.; Morgan, B.; Pham, V.; Holien, J. K.; Parker, M. W.; Thompson, P. E.; Chai, S. Y. 
Identification and development of specific inhibitors for insulin-regulated aminopeptidase as a new class of cognitive enhancers. Br. J. Pharmacol. 2011, $164,37-47$.

6. Diwakarla, S.; Nylander, E.; Gronbladh, A.; Vanga, S. R.; Khan, Y. S.; Gutierrez-de-Teran, H.; Savmarker, J.; Ng, L.; Pham, V.; Lundback, T.; JenmalmJensen, A.; Svensson, R.; Artursson, P.; Zelleroth, S.; Engen, K.; Rosenstrom, U.; Larhed, M.; Aqvist, J.; Chai, S. Y.; Hallberg, M. Aryl sulfonamide inhibitors of insulin-regulated aminopeptidase enhance spine density in primary hippocampal neuron cultures. ACS Chem. Neurosci. 2016, 7, 1383-1392.

7. Borhade, S. R.; Rosenstrom, U.; Savmarker, J.; Lundback, T.; JenmalmJensen, A.; Sigmundsson, K.; Axelsson, H.; Svensson, F.; Konda, V.; Skold, C.; Larhed, M.; Hallberg, M. Inhibition of insulin-regulated aminopeptidase (IRAP) by arylsulfonamides. ChemistryOpen 2014, 3, 256-263.

8. Kokkala, P.; Mpakali, A.; Mauvais, F. X.; Papakyriakou, A.; Daskalaki, I.; Petropoulou, I.; Kavvalou, S.; Papathanasopoulou, M.; Agrotis, S.; Fonsou, T. M.; van Endert, P.; Stratikos, E.; Georgiadis, D. Optimization and structure-activity relationships of phosphinic pseudotripeptide inhibitors of aminopeptidases that generate antigenic peptides. J. Med. Chem. 2016, 59, 9107-9123.

9. Papakyriakou, A.; Zervoudi, E.; Tsoukalidou, S.; Mauvais, F. X.; Sfyroera, G.; Mastellos, D. C.; van Endert, P.; Theodorakis, E. A.; Vourloumis, D.; Stratikos, E. 3,4-diaminobenzoic acid derivatives as inhibitors of the oxytocinase subfamily of M1 aminopeptidases with immune-regulating properties. J. Med. Chem. 2015, 58, 1524-1543. 
10. Stratikos, E. Regulating adaptive immune responses using small molecule modulators of aminopeptidases that process antigenic peptides. Curr. Opin. Chem. Biol. 2014, 23C, 1-7.

11. Zervoudi, E.; Saridakis, E.; Birtley, J. R.; Seregin, S. S.; Reeves, E.; Kokkala, P.; Aldhamen, Y. A.; Amalfitano, A.; Mavridis, I. M.; James, E.; Georgiadis, D.; Stratikos, E. Rationally designed inhibitor targeting antigen-trimming aminopeptidases enhances antigen presentation and cytotoxic T-cell responses. Proc. Natl. Acad. Sci. U. S. A. 2013, 110, 19890-19895.

12. Mpakali, A.; Saridakis, E.; Harlos, K.; Zhao, Y.; Papakyriakou, A.; Kokkala, P.; Georgiadis, D.; Stratikos, E. Crystal structure of insulin-regulated aminopeptidase with bound substrate analogue provides insight on antigenic epitope precursor recognition and processing. J. Immunol. 2015, 195, 28422851.

13. Hermans, S. J.; Ascher, D. B.; Hancock, N. C.; Holien, J. K.; Michell, B. J.; Chai, S. Y.; Morton, C. J.; Parker, M. W. Crystal structure of human insulin-regulated aminopeptidase with specificity for cyclic peptides. Protein Sci. 2015, 24, 190 199.

14. Chen, H.; Noble, F.; Mothe, A.; Meudal, H.; Coric, P.; Danascimento, S.; Roques, B. P.; George, P.; Fournie-Zaluski, M. C. Phosphinic derivatives as new dual enkephalin-degrading enzyme inhibitors: synthesis, biological properties, and antinociceptive activities. J. Med. Chem. 2000, 43, 1398-1408.

15. Kochan, G.; Krojer, T.; Harvey, D.; Fischer, R.; Chen, L.; Vollmar, M.; von Delft, F.; Kavanagh, K. L.; Brown, M. A.; Bowness, P.; Wordsworth, P.; Kessler, B. M.; Oppermann, U. Crystal structures of the endoplasmic reticulum 
aminopeptidase-1 (ERAP1) reveal the molecular basis for $\mathrm{N}$-terminal peptide trimming. Proc. Natl. Acad. Sci. U. S. A. 2011, 108, 7745-7750.

16. Birtley, J. R.; Saridakis, E.; Stratikos, E.; Mavridis, I. M. The crystal structure of human endoplasmic reticulum aminopeptidase 2 reveals the atomic basis for distinct roles in antigen processing. Biochemistry 2012, 51, 286-295.

17. Stratikos, E.; Stern, L. J. Antigenic peptide trimming by ER aminopeptidases--insights from structural studies. Mol. Immunol. 2013, 55, 212219.

18. Nguyen, T. T.; Chang, S. C.; Evnouchidou, I.; York, I. A.; Zikos, C.; Rock, K. L.; Goldberg, A. L.; Stratikos, E.; Stern, L. J. Structural basis for antigenic peptide precursor processing by the endoplasmic reticulum aminopeptidase ERAP1. Nat. Struct. Mol. Biol. 2011, 18, 604-613.

19. Maier, J. A.; Martinez, C.; Kasavajhala, K.; Wickstrom, L.; Hauser, K. E.; Simmerling, C. ff14SB: Improving the accuracy of protein side chain and backbone parameters from ff99SB. J Chem Theory Comput 2015, 11, 3696-3713.

20. Albiston, A. L.; Morton, C. J.; Ng, H. L.; Pham, V.; Yeatman, H. R.; Ye, S.; Fernando, R. N.; De Bundel, D.; Ascher, D. B.; Mendelsohn, F. A.; Parker, M. W.; Chai, S. Y. Identification and characterization of a new cognitive enhancer based on inhibition of insulin-regulated aminopeptidase. FASEB J. 2008, 22, 42094217.

21. Albiston, A. L.; Pham, V.; Ye, S.; Ng, L.; Lew, R. A.; Thompson, P. E.; Holien, J. K.; Morton, C. J.; Parker, M. W.; Chai, S. Y. Phenylalanine-544 plays a key role in substrate and inhibitor binding by providing a hydrophobic packing point at the active site of insulin-regulated aminopeptidase. Mol. Pharmacol. 2010, 78, 600607. 
22. Swain, C. J.; Seward, E. M.; Cascieri, M. A.; Fong, T. M.; Herbert, R.; MacIntyre, D. E.; Merchant, K. J.; Owen, S. N.; Owens, A. P. Identification of a series of 3-(benzyloxy)-1-azabicyclo[2.2.2] octane human NK1 antagonists. J. Med. Chem. 1995, 38, 4793-4805.

23. Stamogiannos, A.; Koumantou, D.; Papakyriakou, A.; Stratikos, E. Effects of polymorphic variation on the mechanism of Endoplasmic Reticulum Aminopeptidase 1. Mol. Immunol. 2015, 67, 426-435.

24. Suhre, K.; Sanejouand, Y. H. ElNemo: a normal mode web server for protein movement analysis and the generation of templates for molecular replacement. Nucleic Acids Res. 2004, 32, W610-614.

25. Georgiadou, D.; Hearn, A.; Evnouchidou, I.; Chroni, A.; Leondiadis, L.; York, I. A.; Rock, K. L.; Stratikos, E. Placental leucine aminopeptidase efficiently generates mature antigenic peptides in vitro but in patterns distinct from endoplasmic reticulum aminopeptidase 1. J. Immunol. 2010, 185, 1584-1592.

26. Lorente, E.; Barriga, A.; Johnstone, C.; Mir, C.; Jimenez, M.; Lopez, D. Concerted in vitro trimming of viral HLA-B27-restricted ligands by human ERAP1 and ERAP2 aminopeptidases. PLoS One 2013, 8, e79596.

27. Saveanu, L.; Carroll, O.; Weimershaus, M.; Guermonprez, P.; Firat, E.; Lindo, V.; Greer, F.; Davoust, J.; Kratzer, R.; Keller, S. R.; Niedermann, G.; van Endert, P. IRAP Identifies an endosomal compartment required for MHC Class I cross-presentation. Science 2009, 325, 213-217.

28. Zervoudi, E.; Papakyriakou, A.; Georgiadou, D.; Evnouchidou, I.; Gajda, A.; Poreba, M.; Salvesen, G. S.; Drag, M.; Hattori, A.; Swevers, L.; Vourloumis, D.; Stratikos, E. Probing the S1 specificity pocket of the aminopeptidases that generate antigenic peptides. Biochem. J. 2011, 435, 411-420. 
29. Mountford, S. J.; Albiston, A. L.; Charman, W. N.; Ng, L.; Holien, J. K.; Parker, M. W.; Nicolazzo, J. A.; Thompson, P. E.; Chai, S. Y. Synthesis, structure-activity relationships and brain uptake of a novel series of benzopyran inhibitors of insulin-regulated aminopeptidase. J. Med. Chem. 2014, 57, 1368-1377.

30. Stratikos, E. Modulating antigen processing for cancer immunotherapy. Oncoimmunology 2014, 3, e27568.

31. Evnouchidou, I.; Berardi, M. J.; Stratikos, E. A continuous fluorigenic assay for the measurement of the activity of endoplasmic reticulum aminopeptidase 1 : Competition kinetics as a tool for enzyme specificity investigation. Anal. Biochem. 2009, 395, 33-40.

32. Cer, R. Z.; Mudunuri, U.; Stephens, R.; Lebeda, F. J. IC50-to-Ki: a web-based tool for converting IC50 to Ki values for inhibitors of enzyme activity and ligand binding. Nucleic Acids Res. 2009, 37, W441-445.

33. Gorrec, F. The MORPHEUS protein crystallization screen. J. Appl. Crystallogr. 2009, 42, 1035-1042.

34. Winter, G. xia2: an expert system for macromolecular crystallography data reduction. J. Appl. Crystallogr. 2010, 43, 186-190.

35. McCoy, A. J.; Grosse-Kunstleve, R. W.; Adams, P. D.; Winn, M. D.; Storoni, L. C.; Read, R. J. Phaser crystallographic software. J. Appl. Crystallogr. 2007, 40, 658674.

36. Murshudov, G. N.; Vagin, A. A.; Dodson, E. J. Refinement of macromolecular structures by the maximum-likelihood method. Acta Crystallogr. D Biol. Crystallogr. 1997, 53, 240-255.

37. Adams, P. D.; Afonine, P. V.; Bunkoczi, G.; Chen, V. B.; Davis, I. W.; Echols, N.; Headd, J. J.; Hung, L. W.; Kapral, G. J.; Grosse-Kunstleve, R. W.; McCoy, A. J.; 
Moriarty, N. W.; Oeffner, R.; Read, R. J.; Richardson, D. C.; Richardson, J. S.; Terwilliger, T. C.; Zwart, P. H. PHENIX: a comprehensive Python-based system for macromolecular structure solution. Acta Crystallogr. D Biol. Crystallogr. 2010, 66, 213-221.

38. Emsley, P.; Lohkamp, B.; Scott, W. G.; Cowtan, K. Features and development of Coot. Acta Crystallog.r D Biol. Crystallog.r 2010, 66, 486-501.

39. Fiser, A.; Sali, A. MODELLER: Generation and refinement of homologybased protein structure models. Macromolecular Crystallography, Pt D 2003, $374,461-491$.

40. Anandakrishnan, R.; Aguilar, B.; Onufriev, A. V. H++ 3.0: automating pK prediction and the preparation of biomolecular structures for atomistic molecular modeling and simulations. Nucleic Acids Res. 2012, 40, W537-541.

41. Case, D. A.; Cheatham, T. E.; Darden, T.; Gohlke, H.; Luo, R.; Merz, K. M.; Onufriev, A.; Simmerling, C.; Wang, B.; Woods, R. J. The Amber biomolecular simulation programs. J. Comput. Chem. 2005, 26, 1668-1688.

42. Wang, J. M.; Wolf, R. M.; Caldwell, J. W.; Kollman, P. A.; Case, D. A. Development and testing of a general amber force field. J. Comput. Chem. 2004, $25,1157-1174$.

43. Jakalian, A.; Jack, D. B.; Bayly, C. I. Fast, efficient generation of high-quality atomic charges. AM1-BCC model: II. Parameterization and validation. J. Comput. Chem. 2002, 23, 1623-1641.

44. Papakyriakou, A.; Spyroulias, G. A.; Sturrock, E. D.; Manessi-Zoupa, E.; Cordopatis, P. Simulated interactions between angiotensin-converting enzyme and substrate gonadotropin-releasing hormone: Novel insights into domain selectivity. Biochemistry 2007, 46, 8753-8765. 
45. Jorgensen, W. L.; Chandrasekhar, J.; Madura, J. D.; Impey, R. W.; Klein, M. L. Comparison of simple potential functions for simulating liquid water. J. Chem. Phys. 1983, 79, 926-935.

46. Hawkins, P. C.; Nicholls, A. Conformer generation with OMEGA: learning from the data set and the analysis of failures. J. Chem. Inf. Model. 2012, 52, 29192936.

47. Morris, G. M.; Huey, R.; Lindstrom, W.; Sanner, M. F.; Belew, R. K.; Goodsell, D. S.; Olson, A. J. AutoDock4 and AutoDockTools4: Automated docking with selective receptor flexibility. J. Comput. Chem. 2009, 30, 2785-2791.

48. Huey, R.; Morris, G. M.; Olson, A. J.; Goodsell, D. S. A semiempirical free energy force field with charge-based desolvation. J. Comput. Chem. 2007, 28, 1145-1152.

49. Salomon-Ferrer, R.; Gotz, A. W.; Poole, D.; Le Grand, S.; Walker, R. C. Routine microsecond molecular dynamics simulations with AMBER on GPUs. 2. Explicit solvent particle mesh Ewald. J. Chem.Th. and Comp.n 2013, 9, 3878-3888. 50. Feenstra, K. A.; Hess, B.; Berendsen, H. J. C. Improving efficiency of large time-scale molecular dynamics simulations of hydrogen-rich systems. J. Comput. Chem. 1999, 20, 786-798.

51. Pastor, R. W.; Brooks, B. R.; Szabo, A. An Analysis of the accuracy of Langevin and molecular-dynamics algorithms. Mol. Phys. 1988, 65, 1409-1419.

52. Berendsen, H. J. C.; Postma, J. P. M.; Vangunsteren, W. F.; Dinola, A.; Haak, J. R. Molecular-dynamics with coupling to an external bath. J. Chem. Phys. 1984, 81, 3684-3690.

53. Darden, T.; York, D.; Pedersen, L. Particle Mesh Ewald - an N.Log(N) Method for Ewald sums in large systems. J. Chem. Phys. 1993, 98, 10089-10092. 
54. Roe, D. R.; Cheatham, T. E. PTRAJ and CPPTRAJ: Software for processing and analysis of molecular dynamics trajectory data. J. Chem. Th. Comp. 2013, 9, 3084-3095.

55. Humphrey, W.; Dalke, A.; Schulten, K. VMD: Visual molecular dynamics. J. Mol. Graph. \& Model. 1996, 14, 33-38. 
Table 1: Crystallographic data and refinement statistics

\begin{tabular}{|c|c|}
\hline PDB entry code & $5 \mathrm{MJ} 6$ \\
\hline Space group & $\mathrm{P} 22_{1} 2_{1} 2_{1}$ \\
\hline Cell & $\begin{array}{l}a=112.24 \AA \\
b=143.17 \AA \\
c=148.99 \AA \\
\alpha, \beta, \gamma=90^{\circ}\end{array}$ \\
\hline \multicolumn{2}{|c|}{ Data collection } \\
\hline Temperature (K) & 100 \\
\hline Resolution (Å) & $\begin{array}{l}40.80-2.53 \\
(2.60-2.53)^{*}\end{array}$ \\
\hline Completeness & $100(100)^{*}$ \\
\hline Redundancy & $13.2(13.1)^{*}$ \\
\hline $\mathbf{R}_{\text {merge }}(\%)$ & $0.199(1.739)^{*}$ \\
\hline $\mathrm{I} / \sigma(\mathrm{I})$ & $11.4(1.6)^{*}$ \\
\hline Unique reflections & 80735 \\
\hline \multicolumn{2}{|l|}{ Refinement } \\
\hline Refinement program & Phenix.refine \\
\hline Resolution $(\AA)$ & 2.53 \\
\hline Unique reflections used & 80655 \\
\hline $\mathbf{R}_{\text {work }}(\%)$ & 17.35 \\
\hline$R_{\text {free }}(\%)$ & 22.90 \\
\hline CC1 $/ 2$ & $0.997(0.451)$ \\
\hline $\begin{array}{l}\text { rmsd from ideal bond lengths } \\
(\AA \AA)\end{array}$ & 0.010 \\
\hline rmsd from ideal angles $\left({ }^{\circ}\right)$ & 1.389 \\
\hline \multicolumn{2}{|l|}{ Ramachandran statistics } \\
\hline $\begin{array}{l}\text { Non-Gly/Pro residues in } \\
\text { most favoured regions }\end{array}$ & $96.41 \%$ \\
\hline $\begin{array}{l}\text { Non-Gly/Pro residues in } \\
\text { additionally allowed regions }\end{array}$ & $3.37 \%$ \\
\hline $\begin{array}{l}\text { Non-Gly/Pro residues in } \\
\text { disallowed regions }\end{array}$ & $0.23 \%$ \\
\hline
\end{tabular}

* values in parentheses are for the outermost shell 
Table of Contents Graphic

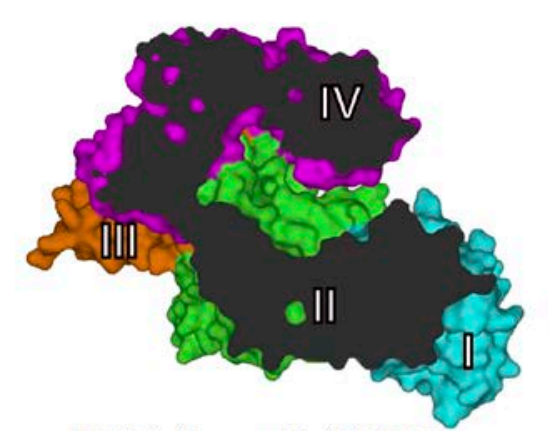

IRAP “open” (5C97)

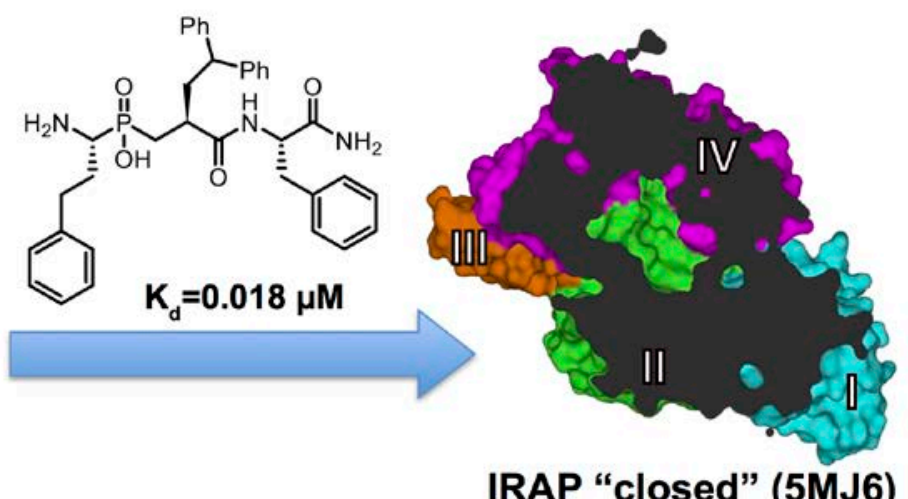

IRAP "closed" (5MJ6) 